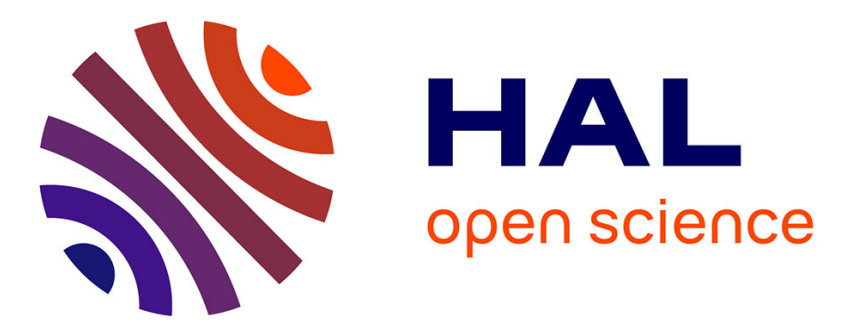

\title{
De l'adoption au rejet d'un commun numérique pour transformer la frontière entre état et citoyens
}

Sébastien Shulz

\section{To cite this version:}

Sébastien Shulz. De l'adoption au rejet d'un commun numérique pour transformer la frontière entre état et citoyens: La trajectoire de la Base Adresse Nationale entre contribution citoyenne, autogouvernement et État-plateforme. Réseaux: communication, technologie, société, 2021, L'action publique au prisme de la gouvernementalité numérique, 225 (1), pp.151-186. 10.3917/res.225.0151. hal-03141725

\section{HAL Id: hal-03141725 \\ https://hal.science/hal-03141725}

Submitted on 15 Feb 2021

HAL is a multi-disciplinary open access archive for the deposit and dissemination of scientific research documents, whether they are published or not. The documents may come from teaching and research institutions in France or abroad, or from public or private research centers.
L'archive ouverte pluridisciplinaire HAL, est destinée au dépôt et à la diffusion de documents scientifiques de niveau recherche, publiés ou non, émanant des établissements d'enseignement et de recherche français ou étrangers, des laboratoires publics ou privés.

\section{다(1)(2)}

Distributed under a Creative Commons Attribution - ShareAlike| 4.0 International 


\section{Version : Pré-print}

Titre : De l'adoption au rejet d'un commun numérique pour transformer la frontière entre État et citoyens. La trajectoire de la Base Adresse Nationale entre contribution citoyenne, autogouvernement et État-plateforme

\section{INTRODUCTION}

À partir de la fin du XVIII siècle, la production d'un vaste ensemble d'informations géographiques sous la forme de listes d'adresses postales, de registres et de cartes rend possible l'avènement de l'administration propre à l'État moderne (Boltanski, 2012 ; Bourdieu, 2015 ; Gardey, 2008 ; Goody, 1979). Elles rendent cognitivement et matériellement possible la gouvernementalité, qu'on peut définir comme un mode spécifique d'exercice du pouvoir des gouvernants sur les gouvernés (Lascoumes, 2004, 2007). Depuis la production par la famille Cassini de la première carte exhaustive de France (Pelletier, 2013), certaines professions au sein d'institutions légitimes produisent l'information géographique officielle.

Pourtant, depuis une quinzaine d'années, cette prétention à produire et imposer une information géographique légitime est remise en cause par l'émergence de nouveaux producteurs issus du monde numérique (Haklay, Singleton et Parker, 2008). Deux typologies d'acteurs se distinguent. D'un côté les plateformes privées comme Googlemaps qui produisent et mettent à disposition, via une offre freemium, des services cartographiques. De l'autre, des associations comme OpenStreetMap (OSM) dont la communauté produit de manière collaborative une carte du monde qu'elle diffuse gratuitement en licence libre. Ces deux types d'acteurs utilisent pleinement les technologies du Web 2.0 en mettant à profit la participation massive de contributeurs (Plantin, 2018). Mais les membres d'OSM critiquent ouvertement Googlemaps car la firme symbolique du capitalisme informationnel garde la propriété et le pouvoir de décision sur les modalités de production et de diffusion des données. À l'inverse, les contributeurs d'OSM conçoivent leur projet comme un commun numérique à l'instar de Wikipedia (Duféal et Noucher, 2017). On définit un commun numérique comme (1) une communauté de producteurs et d'utilisateurs qui gèrent (2) une ressource numérique en vue de son enrichissement dans le temps à travers (3) des règles de gouvernance conjointement élaborées et (4) dont ils protègent le libre accès face aux tentatives d'appropriation exclusive. Malgré leurs différences, ces deux types d'acteurs produisent des données qui ne sont ni officielles, ni souveraines, mais qui sont de plus en plus utilisées, notamment par des acteurs publics 2. Comme l'illustrent les incidents diplomatiques provoqués par des erreurs d'interprétation de Googlemaps par des gardes-frontières (Jacobs, 2012; Staub, 2011), ils déstabilisent le monopole étatique de la production légitime de l'information géographique .

Cette situation de déstabilisation s'est déroulée en France dans le cas d'une information géographique spécifique, l'adresse géolocalisée (voir encadré 1). Au tournant des années 2000, un ensemble d'acteurs publics (IGN, Ministère de la Transition écologique et solidaire, députés), privés (géo-entreprises, gestionnaires de réseaux) et associatifs (OSM, GeoRezo) (voir encadré 1.) se mobilise à travers l'Association Française pour l'information Géographique (AFIGEO) pour réclamer de l'État qu'il produise une base de données numérique regroupant l'ensemble des adresses postales et leurs coordonnées géographiques. Au vu de l'importance historique de cette information pour l'État, on aurait pu s'attendre à ce que des acteurs publics mettent à l'agenda ce problème public et prennent en charge euxmêmes sa production. Pourtant, chose étonnante, un accord est signé en 2015 entre l'IGN, Etalab, La Poste et OSM pour que ce qui deviendra la Base Adresse nationale (BAN) soit produite et gérée comme un commun numérique.

\footnotetext{
Googlemaps revendique un milliard d'utilisateurs mensuels en 2018. Source : https://www.journaldunet.com/ebusiness/publicite/1211137-les-20-chiffres-de-google/. OSM annoncent six millions d'utilisateurs et environ 45000 contributeurs actifs en 2019. Source : https://wiki.openstreetmap.org/wiki/Stats
} 
La première énigme à laquelle je tenterai de répondre est de comprendre pourquoi des acteurs publics ont décidé d'adopter la forme d'un commun numérique pour produire la BAN ? Pourquoi ne pas l'avoir pris en charge eux-mêmes (comme au Danemark) ou privatisé (comme en Angleterre) ? C'est d'autant plus étonnant que la BAN est, à notre connaissance, une des premières expérimentations d'un commun numérique mis en place par un État. La deuxième énigme qui découle de la première tient aux conséquences d'un tel mélange des genres sur l'exercice pratique du pouvoir étatique. Qu'est-ce que la forme du commun numérique vient déplacer au sein de l'administration et dans la relation entre l'État et les citoyens ? Et plus spécifiquement, comment cette nouvelle forme d'action publique reconfigure l'exercice du pouvoir informationnel de l'État en permettant ou en empêchant certains acteurs de produire et d'utiliser d'une certaine manière une information publique ? J'ai entamé cette recherche en faisant l'hypothèse que l'adoption par l'administration de la forme du commun numérique, de ses «principes actifs»- pratiques contributives, mode d'organisation démocratique, etc. - allait transformer l'exercice du pouvoir informationnel de l'État en général, et la relation État/citoyens en particulier, en donnant plus d'autonomie aux communautés numériques productrices d'information, en rendant cette production d'informations officielles perméable aux contributions citoyennes devenues plus légitimes et en transformant le droit afin de protéger juridiquement le libre accès aux ressources ainsi produites contre toute forme d'appropriation exclusive. Cette transformation pourrait s'opérer par l'importation de représentations, pratiques et instruments spécifiques aux communs numériques à travers l'action d'entrepreneurs de cause multipositionnés. Je qualifie cette hypothèse "d'infusion institutionnelle" après Bréthaut et Nahrath (2010).

Dans cet article, je défends d'abord l'argument que la forme du commun numérique a été adoptée pour la BAN parce qu'elle permettait, dans une fenêtre d'opportunité, de traduire et mettre en œuvre le projet réformateur d'un acteur public. Initialement, la question d'une base adresse nationale émerge classiquement comme un problème public dont les différents acteurs en appellent à l'État. Un hiatus, que je nomme avec Dominique Linhardt une "épreuve d'État", s'opère puisque pendant plus de quinze ans, aucun acteur public ne va produire cette base de données tant attendue. Les membres d'OSM, encore marginaux sur la scène de l'information géographique, s'engagent alors dans l'épreuve et provoquent l'État en construisant une base adresse nationale ouverte (BANO) sous la forme d'un commun numérique à partir d'un «hack» de certaines données publiques (I.). Henri Verdier, à la tête de la Direction interministérielle du numérique (DINSIC), saisit l'occasion. Il recrute le président d'OSM au sein de la DINSIC et traduit ces différentes interpellations en faisant appel à la forme assez plastique des communs numériques pour former des alliances et tenter de mener à bien son projet de réforme de l'État par le numérique (II.).

Mon second argument est que le processus de mise en œuvre de la BAN comme commun numérique a soulevé des épreuves - de statut, d'organisation et juridico-économique - pour reconfigurer la relation État/citoyens (III.). Mais leur résolution est systématiquement passée par un abandon de la forme du commun numérique, du partenariat avec OSM et de la participation citoyenne, au profit d'une vision plus souveraine, administrative et pro-marché de la BAN (IV.). Je montre dans la discussion que c'est la forme de l'État-plateforme qui l'a emporté sur celles de l'auto-gouvernance et de la contribution citoyenne, plus proches de "l'esprit" des communs numériques. J'invalide donc en partie l'hypothèse de l'infusion institutionnelle (Bréthaut et Nahrath, 2010). Mais si l'adoption de la forme commun numérique pour la BAN n'a pas encapacité les citoyens dans la production d'informations publiques, elle n'a pas non plus conduit à un désengagement de l'État. Le cas de la BAN montre que les acteurs publics affirment la présence numérique de l'État en gardant la main sur la production et la diffusion de certaines infrastructures informationnelles qui sont, comme le montre Sandra Braman (2009), les instruments de nouvelles formes d'exercice du pouvoir (V.). 
Mon travail s'inscrit dans la vaste littérature qui cherche à décrire et analyser ce que l'adoption de pratiques et de dispositifs numériques font à l'État (Alauzen, 2019; AriVeikko, 2008 ; Goëta, 2016 ; West, 2005). Prenant au sérieux les discours techno-optimistes, une partie de cette littérature s'est penchée sur les transformations de la relation État/citoyen induites par l'adoption des technologies du web 2.0 par les administrations. Le passage du "egovernment" au "we-government" permettrait de dépasser le paradigme du new public management (NPM) pour donner un rôle plus important aux citoyens connectés dans la "coproduction des services publics" (Linders, 2012; Lips, 2012). Linders (2012) établit trois catégories utiles pour décrire ces nouvelles formes de co-production. Le citizen sourcing, où les citoyens aident l'administration à être plus efficace, notamment en contribuant à lui remonter des informations (ex. Fixmystreet). Le government as a Platform, où l'État rend disponible en libre accès une partie de son infrastructure informationnelle pour permettre à la société civile de proposer de nouveaux services (ex. data.gouv.fr). Et le Do it Yourself Government, qui met l'accent sur les formes d'auto-organisation des citoyens et où l'État n'a, au mieux, qu'un rôle de facilitateur (ex. OpenStreetMap). Pourtant, deux problèmes se posent avec cette littérature pour éclairer nos énigmes. D'une part, la BAN, en tant que commun numérique mis en place par l'État, n'est pas facile à catégoriser dans la typologie de Linders pourtant très claire théoriquement puisqu'elle pourrait relever des trois catégories à la fois. Contribution citoyenne, auto-organisation et ressource en libre accès sont en effet trois caractéristiques des communs numériques tels que je les ai défini plus haut. D'autre part, cette littérature reste tributaire des discours techno-optimistes et certains techno-critiques redoutent que les formes numériques de co-production ne soient en réalité qu'un prétexte pour accélérer le désengagement de l'État (Bates, 2014 ; Franceschetti, 2016). Entre ces deux positions, encore peu d'enquêtes empiriques ont permis de saisir tous les enjeux de ces modalités de coproduction, notamment les résistances et les déplacements qu'elles engagent. La BAN semble préfigurer a priori une reconfiguration de la relation État/société en donnant plus de pouvoir de production informationnelle aux citoyens, mais seule une plongée dans sa mise en œuvre nous permettra d'en saisir les effets concrets.

J'ai donc mené l'enquête à travers une série de vingt-trois entretiens auprès de l'ensemble des acteurs publics, associatifs et privés concernés par l'adresse en Frances', l'analyse d'un corpus varié (billets de blogs, rapports, articles, textes de loi, tweets) et plusieurs terrains lors de réunions de travail et de journées de présentation de la BAN. J'appuie ma démonstration en combinant les concepts développés par une partie de la sociologie politique des réformes de l'État (Bezes et Le Lidec 2011; Lascoumes et Le Galès 2012) à une sociologie pragmatique s'intéressant aux mises en doute de l'État et attentive à l'échelle fine des dispositifs sociotechniques pour y déceler les épreuves qui s’y jouent (Linhardt, 2009, 2012).

\section{Encadré 1. L'adresse géolocalisée : une information élusive et un écosystème varié}

L'adresse peut être définie comme l'identification d'une entité physique (boite aux lettres, entrée d'un bâtiment, etc.) par des informations nominatives et/ou sa localisation géographique (coordonnées $\mathrm{x}, \mathrm{y}$ ). Elle a différentes fonctions comme localiser un endroit pour s'y rendre ou l'identifier à des fins administratives. Chaque acteur privilégie des types d'adresses différents. Dans un même bâtiment, La Poste cherche la localisation des boîtes aux

${ }^{3}$ Par institution : IGN (ancien directeur, responsable technique de la BAN et deux autre membres), La Poste (deux commerciaux du Service National de l'Adresse), Direction Inter-ministeriel du Numérique et de la Communication (ancien directeur, responsable de la BAN et fondateur de la BANO), Direction générale des Finances Publiques (responsable de la cellule topographique du Cadastre, son assistant technique et son assistant juridique), Assemblée nationale (députée rédactrice du rapport sur la donnée géographique souveraine de 2018), Gendarmerie nationale (responsable et assistant de la Section Information Géographique Opérationnelle), Autorité de Régulation des Communications Électroniques et des Postes (conseillé du président), OpenStreetMap (président d'OSM France et membre d'OSM Royaume-Uni responsable de l'adresse), service départemental d'incendie et de secours du 91 (chef du service cartographique), mairies (maire de la ville de Rully et responsable des données de la ville de Paris), Direction régionale de l'Environnement, de l'Aménagement et du Logement de Normandie (responsable de l'information géographique et de l'open data), Association française de l'information géographique (porte-parole) et finalement ancien responsable de la base adresse du Danemark. 
lettres et les secouristes la localisation des sorties de secours. Finalement, l'adresse peut s'inscrire sur différents supports informationnels (voir figure 1.)

\section{Figure 1. Le 5 rue de Tombouctou (75018) dans tous ses états}

1. Image IGN

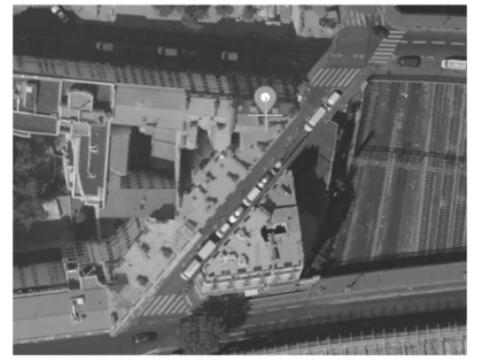

2. Plan vectorisé OSM

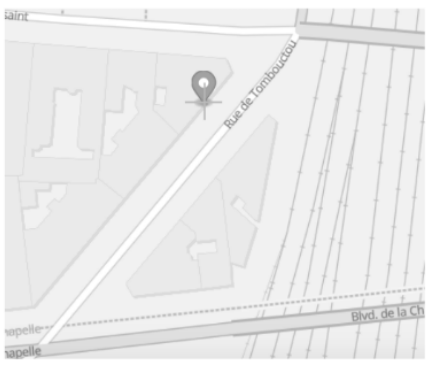

4. Extrait du fichier CSV BAN
3. Plan du cadastre

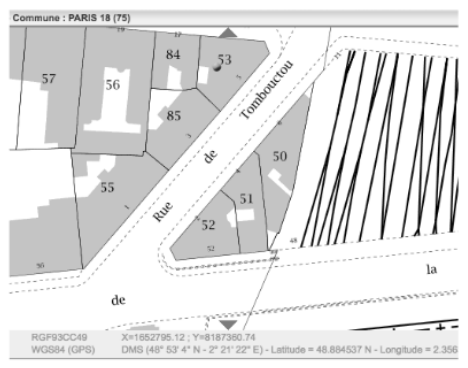

\begin{tabular}{|c|c|c|c|c|c|c|c|c|}
\hline id & id_fantoir & numero & nom_voie & code_postal & code_insee & nom_commi code_insee_. & & y \\
\hline 75118_9344. & 75118_9344 & 1 & Rue de Tombouctou & 75018 & 75118 & Paris $18 \mathrm{e}$ Arrondissement & 652786.18 & 6865137.55 \\
\hline 75118_9344. & 75118_9344 & 2 & Rue de Tombouctou & 75018 & 75118 & Paris $18 \mathrm{e}$ Arrondissement & 652802.36 & 6865135.03 \\
\hline 75118_9344. & 75118_9344 & 3 & Rue de Tombouctou & 75018 & 75118 & Paris $18 \mathrm{e}$ Arrondissement & 652800.35 & 6865153.88 \\
\hline 75118_9344. & 75118_9344 & 4 & Rue de Tombouctou & 75018 & 75118 & Paris $18 \mathrm{e}$ Arrondissement & 652811.63 & 6865145.76 \\
\hline $75118 \quad 9344$ & 751189344 & 5 & Rue de Tombouctou & 75018 & 75118 & Paris $18 \mathrm{e}$ Arrondissement & 652811.88 & 6865167.25 \\
\hline 75118_9344. & 75118_9344 & 6 & Rue de Tombouctou & 75018 & 75118 & Paris $18 \mathrm{e}$ Arrondissement & 652821.18 & 6865156.82 \\
\hline
\end{tabular}

Lecture : Les deux premières images (1.2.) sont extraites de l'outil de visualisation de la BAN (source : adresse.data.gouv fr/map). La troisième (3.) provient des planches cadastrales mises en ligne par la Direction générale des Finances publiques. La dernière (4.) est l'extrait du fichier de la BAN (source : adresse.data.gouv.fr/data/ban/adresses/latest/csv/ consulté le 20/01/2020).

L'écosytème de l'adresse en France est hétérogène et composé d'acteurs publics, privés et associatifs4. Voici leurs caractéristiques.

- Les mairies sont seules dépositaires de l'autorité sur l'adresse sémantique : elles peuvent créer ou modifier les noms et numéros de voie. On estime à 2 millions le nombre d'habitations qui n'ont pas d'adresse sémantique (généralement des lieuxdits) et tous les ans plus de 400000 bâtiments sont détruits, modifiés ou construits ce qui génère un besoin constant de mise à jour des adresses.

- La Direction générale des finances publiques (DGFiP) entretient pour les besoins de recouvrement de l'impôt une base de données regroupant le nom de la voie et le numéro associé sur les parcelles cadastrales géographiques.

- L'Institut national de la statistique et des études économiques (INSEE) a constitué le Répertoire des Immeubles Localisés, une base d'adresses géolocalisées dans le cadre de ses travaux de recensement pour les villes de plus de 10000 habitants.

- Le Service National de l'Adresse (SNA) de La Poste est créé en 1996 pour permettre l'automatisation du tri postal. Il constitue en interne le Référentiel de l'Adresse National pour s'assurer de la correspondance avec les adresses sémantiques écrites sur les plis postaux (encore aujourd'hui, environ 300 millions de plis par an sont renvoyés à l'expéditeur, ce qui génère de nombreux coûts). La Poste, société anonyme à capitaux publics depuis 2010 , vend des services aux entreprises pour leur permettre de normaliser leurs bases adresses internes (de clients notamment).

- L'IGN, acteur historique de l'information géographique, a été mandaté par l'État (suite au rapport Langagne de 1999) pour constituer un Référentiel à Grande Échelle dont une base de données adresse (DB Adresse). Cette dernière, créée en 2004, est toujours en production.

D'autres acteurs producteurs ou usagers de la donnée adresse à noter

- Les services départementaux d'incendie et de secours (SDIS) et la police nationale

‘ Pour plus de détail, voir Groupe de Travail Adresse (2011) Pour une politique nationale de la donnée Adresse. December. AFIGEO 
qui ont besoin d'une adresse fiable pour l'identification des appels et l'envoi des secours développent en interne des bases adresse.

- Les gestionnaires de réseaux (ERDF, les opérateurs télécoms) sont consommateurs de la donnée adresse pour assurer le raccordement des usagers.

- Les géo-entreprises (producteurs de données, éditeurs de logiciels et développeurs d'applications, consultants en géomatiques, médias spécialisés) sont plus de 500 en France.

- L'économie numérique (Uber, Airbnb, Leboncoin, etc.) est consommatrice de bases de données d'adresses géolocalisées.

- Un ensemble d'associations et de réseaux dont les plus importantes sont l'Association française pour l'information géographique (AFIGEO), OpenStreetMap et le portail GeoRezo.

\section{ARCHÉOLOGIE DE LA BAN : MISE EN PLACE DE L'ÉPREUVE D’ÉTAT}

Au tournant des années 2000, des doutes s'élèvent quant à la place et au rôle de l'État par rapport aux nouveaux acteurs numériques de l'information géographique. Pendant quinze ans, il fait l'objet d'interpellations externes et internes à l'administration. Dans les années 2010 ces interpellations convergent pour réclamer de l'État qu'il produise et diffuse une base nationale d'adresses géolocalisées. Pourtant, aucun acteur public ne répond «au nom de l'État » et ce hiatus constitue ce que je qualifie d'épreuve d'État (Linhardt, 2018). La communauté OpenStreetMap, et en particulier son président de l'époque Christian Quest, entrent dans cette épreuve d'État en décidant de produire eux-mêmes une base adresse nationale ouverte (BANO) en « hackant » des données publiques.

\section{Critiques et interpellations de l'État (1999-2010)}

Trois types d'acteurs se mobilisent pour que l'État français produise et diffuse une base d'adresses géolocalisée : l'Union européenne, les militants de l'open data et des réformateurs au sein de l'administration.

Dans les années 2000, "la donnée" commence progressivement à être définie par les cabinets de conseils, les journalistes économiques et l'Union européenne comme le prochain "carburant de la nouvelle économie numérique" (Chignard et Benyayer, 2015). Ces années-là connaissent une explosion des services et des promesses technologiques autour de la numérisation de l'information géographique qui semble transformer assez radicalement ce secteur (Denègre et Salgé, 2004). L'UE joue un rôle important dans la discussion autour de l'information géographique ". En 2007, elle vote la directive INSPIRE qui concerne spécifiquement la mise à disposition de l'information géographique publique dont l'adresse géolocalisée (voir annexe 1 de la directive). Elle vise à créer, dans une perspective de croissance économique, une infrastructure des données adresses de l'Union européenne en harmonisant l'ensemble des bases nationales et régionales.

L'autre courant qui se mobilise pour la mise à disposition d'une base adresse s'inscrit dans le mouvement de l'open data. Des militants, des juristes ou encore des entrepreneurs critiquent l'administration en considérant qu'elle garde la main sur une information publique pourtant produite aux frais du contribuable (Goëta 2016). La défaillance des États à fournir

\footnotetext{
' Parmi les 10 principales sources qui utilisent la notion de «big data » entre 2000 et 2010 les sources de presse économiques sont majoritaires. Source : Factiva

, Voir notamment la directive SPI concernant la mise à disposition d'informations publiques (Parlement européen, 2003).

${ }^{10}$ Les deux principales sources des textes qui mentionnent la notion d' «information géographique » sur Factiva depuis 1994 sont l'Union et la commission Européennes. Source : Factiva
} 
gratuitement une information géographique qu'ils produisent et dont ils ont le monopole est d'ailleurs à l'origine du projet d'OSM.

Les interpellations sont également émises de l'intérieur de l'administration. Tout au long des années 2000, de nombreux députés, conseillers et hauts fonctionnaires discutent par rapports interposés de la manière dont l'État doit adopter les pratiques et les outils du numérique (Bellon, 2018). Sur la question de l'adresse, l'ensemble des rapports défendent l'idée que l'État doit mettre en place une base adresse nationale qui deviendrait un référentiel commun à l'ensemble des acteurs publics ${ }^{13}$. Trois justifications sont apportés. D'une part stimuler l'activité économique. D'autre part rendre l'action publique plus efficace. Il existe plus d'une dizaine de bases adresses dans des silos administratifs (voir encadré 1) leur mutualisation permettrait de réduire les dépenses publiques et les risque d'erreurs. Finalement, rendre l'action publique plus souveraine. Les missions régaliennes de l'État sont tributaires d'une adresse géolocalisée, et les rapports mettent en avant les risques qu'il y aurait à baser des décisions publiques sur la base d'informations étrangères ou qui ne soient pas certifiées officiellement ${ }^{4}$.

\section{Une réunion, un rapport, une convergence des revendications et un État sans voix (2010-} 2013)

Début 2010 l'AFIGÉO organise dans le siège de La Poste une réunion sur la thématique de l'adresse. On y retrouve plus de cent vingt acteurs qui représentent les différents courants que j'ai évoqués ci-dessus (voir encadré 1.). Voici comment se conclut cette journée : «Pour la constitution d'un référentiel Adresse national (...) la désignation d'un maître d'ouvrage est nécessaire et ce rôle devrait être assuré par l'État ». (AFIGEO, 2010). À la suite de cette réunion, un groupe de travail adresse est monté et mené par l'Association Française pour l'information Géographique. Il est composé de membres de l'IGN, de La poste, de SDIS, de communautés d'agglomération et de différents ministères dans le but de produit le rapport Pour une politique nationale de la donnée Adresse (Groupe de Travail Adresse, 2011) remis au Premier ministre. Et ce rapport, de nouveau, ré-insiste sur la nécessité que l'État prenne en charge la constitution d'une base de données adresse unifiée.

Tableau 1. Récapitulatif

\begin{tabular}{|c|c|c|c|}
\hline Acteurs & Revendication & $\begin{array}{l}\text { Figure } \\
\text { d'Etat }\end{array}$ & $\begin{array}{l}\text { Horizon } \\
\text { d'action }\end{array}$ \\
\hline $\begin{array}{c}\text { Union } \\
\text { européenne, } \\
\text { Cabinets de } \\
\text { conseil }\end{array}$ & $\begin{array}{l}\text { L'État doit fournir une infrastructure } \\
\text { informationnelle sur l'adresse pour } \\
\text { stimuler l'activité économique }\end{array}$ & État libéral & \multirow{4}{*}{$\begin{array}{c}\text { L'État doit } \\
\text { produire et } \\
\text { diffuser } \\
\text { gratuitement une } \\
\text { base adresse } \\
\text { nationale }\end{array}$} \\
\hline $\begin{array}{c}\text { Militants, } \\
\text { juristes, } \\
\text { entrepreneurs }\end{array}$ & $\begin{array}{l}\text { L'Etat doit rendre gratuitement accessibles } \\
\text { des données qu'il produit avec de l'argent } \\
\text { public }\end{array}$ & État redevable & \\
\hline \multirow{2}{*}{$\begin{array}{l}\text { Députés, haut- } \\
\text { fonctionnaires, } \\
\text { réformateurs }\end{array}$} & $\begin{array}{l}\text { L'Etat doit devenir plus efficace tout en } \\
\text { rationalisant les dépenses publiques grâce } \\
\text { aux outils numériques }\end{array}$ & État efficace & \\
\hline & $\begin{array}{l}\text { L'Etat doit avoir la maîtrise de la } \\
\text { production des informations sur lesquelles } \\
\text { il base ses missions régaliennes }\end{array}$ & État souverain & \\
\hline
\end{tabular}

Tout au long des années 2000, l'État est ainsi interpellé à différentes reprises et de différentes manières. Mais plus l'expression de la nécessité d'une base d'adresse nationale se fait pressante, plus l'absence de prise en compte du problème au sein de l'État devient

${ }^{13}$ rapport Langagne (1999), rapport de la Commission Nationale de l'Information Géographique (2002), rapport du Conseil Général des Technologies de l'Information (2007), rapport du Conseil de l'orientation de l'édition publique (2012).

${ }^{14}$ «La cartographie a été historiquement considérée comme un attribut de souveraineté. (...) La dépendance informationnelle serait le premier pas vers la dépendance économique et politique » (Langagne 1999) 
problématique. Pendant presque quinze ans, l'État ne réagit pas. Il serait plus précis de dire qu'aucune administration ne répond «au nom de l'État». À cette époque, les deux acteurs pressentis pour prendre en charge la BAN, à savoir l'IGN et La Poste, vendent des bases adresses, ne souhaitent pas voir l'apparition d'une base adresse publique et gratuite (voir partie III) et sont en accord avec la doctrine administrative de l'époque qui repose sur la valorisation économique du patrimoine immatériel de l'État et non sa gratuité (Goëta, 2016). L'État reste ainsi sans voix faute de porte-parole. Nous sommes ici dans la première étape du processus d'épreuve d'État que conceptualise D. Linhardt: un problème public apparaît, certains pensent que l'État devrait intervenir d'une telle manière et il ne le fait pas (Linhardt, 2018).

Selon D. Linhadrt, il arrive qu'un "processus d'étatisation" s'en suive, c'est-à-dire qu'après l'avoir mis sur l'agenda public, l'État prenne en charge officiellement (par l'inscription dans le droit par exemple) le problème public en question. Or j'ai rappelé en introduction que dans le cas de la BAN, ce processus d'étatisation a pris une forme particulière. Comment est-on passé de cette situation bloquée à la signature du partenariat pour la création de la BAN sous la forme d'un commun numérique?

\section{OpenStreetMap s'engage dans l'épreuve : le « hack » du plan cadastral (2013)}

L'apparition d'un nouvel acteur - OpenStreetMap (OSM) - et l'action de l'entrepreneur de réforme C. Quest, s'avèrent centrales pour l'explication. OSM est un projet de cartographie participative lancée en 2004. La communauté française est l'une des plus actives et

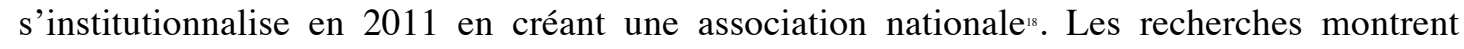
qu'une part importante des contributeurs d'OSM France considèrent OSM comme un commun numérique et qu'ils font partie de l'écosystème de l'information géographique (voir encadré 1.) (Duféal, Jonchères et Noucher, 2016; Duféal et Noucher, 2017). C. Quest, le vice-président de l'association à cette époque, se rappelle qu'en 2012 :

« des gens bien implantés dans le milieu de géographie française (...) nous ont parlé des adresses. Entre autres, il y avait l'AFIGEO (...) qui était assez remonté à l'époque, parce qu'il y avait eu tout un travail qui avait été fait (...) ça faisait un an que le rapport était sorti et il ne s'était absolument à rien passé. » (C. Quest, octobre 2017)

OSM, un acteur encore marginal sur la scène de l'information géographique fait alors irruption dans la dynamique du problème public. C. Quest et quelques autres membres d'OSM décident de produirent eux-mêmes une Base Adresse nationale Ouverte (BANO). Ils réalisent la première version de la BANO au moyen d'un « hack» (bidouillage) technique. Grâce à un script informatique, ils «scrapent" » les millions de planches cadastrales fournies en ligne en format PDF par la Direction générale des finances publiques (DGFiP). En quelques mois ils récoltent et «nettoient », grâce aux remontés de la communauté, plus de 15 millions d'adresses géolocalisées qui passent d'informations géographiques inertes sur PDF à des données numériquement exploitables dans une base de données en format CSV. Le projet BANO suscite un engouement et donne une légitimité à l'association auprès des acteurs publics.

«On voyait débarquer (...) plein d'acteurs institutionnels qui étaient intéressés (...). On se retrouvait en position de lobbying et à mettre un coup de pied dans une fourmilière professionnelle et institutionnelle » (Vincent de Château-Thierry, président d'OSM France, octobre 2018)

Les membres de l'association OSM France et C. Quest en particulier mettent ainsi en place une base de données que l'État devait produire. Ils le font de manière analogue à leur propre forme sociotechnique : celle du commun numérique. La BANO est en effet le projet (1) d'une

\footnotetext{
${ }^{18}$ La France compte un grand nombre de contributeurs par habitants par rapport à d'autres pays. Source : https://www.openstreetmap.org/stats/data_stats.html

is Terme qu'on peut définir comme une technique d'extraction du contenu de sites Web, via un programme, dans le but de le transformer pour permettre son utilisation dans un autre contexte.
} 
communauté de producteurs/usagers qui gèrent (2) la ressource en vue de son enrichissement via (3) une gouvernance souple qui passe principalement par des listes de diffusion et (4) qui est diffusée sous licence OdBL pour la protégée juridiquement des réappropriations exclusives. Cette étape, cruciale, n'est pourtant pas encore suffisante pour expliquer la raison pour laquelle des acteurs publics se saisissent de cette occasion pour réformer l'État par les communs numériques. Il nous faut maintenant pousser les portes de l'administration.

\section{RÉFORMER L’ÉTAT PAR LES COMMUNS NUMÉRIQUES}

J'ai décrit jusqu'ici ce que P. Bezes et P. Le Lidec nomment des "conditions génératives" d'une réforme : revendications exogènes et endogènes de transformation numérique de l'État, émergence d'un nouvel acteur dans une arène publique et remise en cause de l'ordre institutionnelle. Comment est-on passé de ces conditions génératives à la volonté de réformer l'État par les communs numériques? Henri Verdier, joue ici le rôle de déclencheur en tant qu' « entrepreneur de réforme » (Bezes et Le Lidec, 2011).

Encart 2. Le parcours d'un entrepreneur de réforme

Le parcours d'Henri Verdier permet d'éclairer la manière dont il parvient à concilier des points de vue disparates autour d'une vision commune des communs numériques. Fils d'une famille de fonctionnaires, il entre à l'École normale supérieure et suit en parallèle des études de sociologie politique. Il cofonde entre 1995 et 2012 deux entreprises dans le secteur numériques. Au tournant des années 2000, il s'acculture aux logiciels libres en fréquentant la section numérique du Parti Socialiste Temps réels (Beauvallet et Ronai, 2005). Puis à partir des années 2010 il se nourrit des enjeux du numérique à l'institut Télécom où il monte le think tank Futur numérique et se forme à l'économie des plateformes à l'occasion de voyages d'études internationaux (à la Sillicon Valley notamment). Il est également membre fondateur du pôle de compétitivité Cap digital où il «prend conscience de la force d'un écosystème » en mettant en place des projets de coopération entre start-ups, grandes entreprises et acteurs publics locaux (Entretien avec H. Verdier, janvier 2020). Il est nommé à la tête d'Etalab en 2013, premier Administrateur général des données de l'État en 2014 puis directeur de la DINSIC en 2015.

On voit qu'il est acculturé, comme la plupart des entrepreneurs de réforme, à plusieurs milieux sociaux, dont trois particulièrement important ici, ceux : du logiciel libre, de l'économie numérique et de l'administration publique.

\section{De la réforme par le numérique à la réforme par les communs numériques}

H. Verdier est nommé en 2013 à la tête d'Etalab. Il entre dans l'administration avec un objectif : « [il faut] d'abord et avant tout une transformation de l'État lui-même : une remise en cause des formes actuelles de la bureaucratie » (Verdier, 2018). Comme l'analyse P. Bezes, depuis plusieurs décennies l'administration bureaucratique «est devenue le problème » des réformateurs de l'État (Bezes, 2009, p. 29). À la lecture des nombreuses interventions publiques d'H. Verdier, on peut dégager trois axes privilégiés pour mener à bien sa réforme de l'État.

D'abord, l'administration doit parvenir à inventer des modes organisationnels plus perméables et ouverts à l'extérieur de l'administration. Comme il l'indique dans son ouvrage, s'ouvrir à la «force de la multitude » semble être une clé du succès des grandes plateformes numériques dont l'État pourrait s'inspirer. Cette dimension renvoie à la forme de coproduction du citizen sourcing. Ensuite, l'administration doit accepter des modes de gestion par projet plus agiles inspirés du monde du développement informatique (à la fois des startsup et du logiciel libre), en laissant une place plus grande à l'autonomie des agents vis-à-vis de 
leur hiérarchie. Cela s'apparente à la forme du do-it-yourself government. Mais parallèlement, l'administration et les citoyens doivent rester souverains sur leur infrastructure informationnelle pour ne pas dépendre des plateformes privées comme Googlemaps ${ }^{\star 2}$. L'État doit être lui-même une plateforme.

Ouverture à la contribution des citoyens, autonomie des agents contre les lourdeurs bureaucratiques et souveraineté face aux GAFA : ce triptyque, aux colorations à la fois des communs, libérale et étatiste constitue la trame d'un récit réformateur porté par H. Verdier suffisamment large et plastique pour qu'un ensemble d'acteurs appartenant à des mondes sociaux distincts s'y reconnaissent. Lorsqu'H. Verdier prend connaissance du projet de la BANO, il y voit une fenêtre d'opportunité. La forme sociotechnique des communs numériques semble correspondre à son triptyque et peut constituer un instrument efficace et fédérateur pour entreprendre sa réforme numérique de l'État.

\section{Les communs numériques comme traducteur d'intérêts divergents}

Pour mener à bien sa réforme, il cherche à trouver des alliés et à éviter le blâme. Il commence par s'allier avec OSM France en recrutant C. Quest courant 2014 afin que ce dernier développe le projet BANO au sein de l'administration. Pour Quest, l'autonomie qui lui est accordée au sein d'Etalab, l'importance affichée par cette agence à l'ouverture à la contribution citoyenne et l'équipe composée de nombreux défenseurs des logiciels libres l'enrôlent dans le projet réformateur d'H. Verdier ${ }^{23}$. Son rôle, en tant qu'acteur multipositionné entre différentes univers sociaux (associatif, hacker, géomatique, administratif), semble crucial pour importer - et légitimer - la forme du commun numérique au sein de l'administration. Mais rapidement, le sujet devient politique.

« J'ai commencé à parler de BANO2 : base adresse nationale ouverte et officielle. À ce moment-là ça, je suis clairement allé marcher sur les plates-bandes de l'IGN » (C. Quest, 2018, je souligne)

H. Verdier parvient alors à nouer une alliance avec l'IGN. L'affaire n'est pas jouée d'avance parce que cet établissement public administratif selon les textes, devenu largement commercial (40\% de ses fonds viennent de ses ventes) commercialise une base adresse. Mais l'ancien directeur de l'IGN, polytechnicien ayant passé la plus grande partie de sa carrière dans des «instituts hybrides public-privé » partage avec H. Verdier la conviction qu'il faut «appliquer un certain nombre de méthodes du privé, des modes de managements (...) à des missions de services publics » (juillet 2018). Il partage également une méfiance à l'égard des GAFA et mesure les risques d'une perte de souveraineté sur la donnée géographique. Cet ensemble d'éléments le convainc finalement de rejoindre le partenariat :

«C'était une réflexion à la fois globale sur l'État et d'une réflexion de fond sur les missions de l'IGN entre les GAFA et le collaboratif. C'est ce qui a amené d'une base adresse interne et commercialisée à un common géré de manière étatique et mis à disposition de tout le monde » (ancien directeur de l'IGN, juillet 2018)

Pour le Service National de l'Adresse (SNA) de La poste, l'intérêt d'entrer dans le projet repose à l'époque sur les bénéfices d'une mise en commun des efforts de production et de maintenance d'une base de données coûteuse à entretenir ${ }^{4}$. Comme s'en rappelle un directeur commercial du SNA :

\footnotetext{
"Ainsi m'explique-t-il, «le comparable avec Google c'est la Compagnie des Indes. Ils sont trans-étatiques, plus forts que bien des États, et capables d'infléchir (il tape sur la table plusieurs fois) le destin des États à leur avantage. » (janvier 2020).

${ }^{23}$ Comme il l'écrira dans sa lettre de démission : «J'ai rejoint la mission Etalab en 2014. Si j'ai accepté cette offre, c'est parce j'y retrouvais des valeurs essentielles : ouverture, transparence, approche disruptive, gouvernance horizontale, internalisation des compétences, vision à long terme. Cette mission rattachée au premier ministre était à ma connaissance une des rares administrations réellement ouverte sur le monde extérieur (...) » (archive, 21 février 2019)

${ }^{24}$ Plus d'une trentaine de salariés de ce service de La Poste maintiennent la base adresse en interne.
} 
«C'est Etalab et l'IGN qui nous ont proposé d'entrer dans la création de la BAN. On a évidemment réfléchi très fortement à la question, et puis on s'est embarqué en se disant que c'était intéressant parce qu'avoir une base nationale partagée par tous c'est très bien. (...) On a donc signé, tout en sachant qu'on a une activité commerciale et d'exploitation de nos référentiels qui ... nous oblige à demander une situation particulière sur la BAN. »

Finalement, H. Verdier obtient l'aval de son supérieur hiérarchique, Thierry Mandon, alors promu secrétaire d'État à la Réforme de l'État et à la Simplification (SGMAP). Ce dernier vient de publier un rapport intitulé Le numérique pour transformer l'État et dans lequel le discours réformateur d'H. Verdier concernant la BAN vient faire écho.

On voit déjà apparaître des différences de justifications entre les acteurs embarqués, mais le récit réformateur de la $\mathrm{BAN}$ comme commun numérique est assez souple pour permettre de traduire plusieurs perspectives (Akrich, Callon et Latour, 2013). Un premier accord de principe est signé fin 2014 entre La Poste, l'IGN, Etalab et OSM, puis un partenariat officiel est finalement inauguré le 15 avril 2015 dans les locaux de la fondation Mozilla. Le choix de ce lieu, à l'extérieur de l'administration et accueilli par une fondation qui défend les logiciels libres et l'Internet comme bien commun, n'est pas anodin. La constitution de la BAN prenant la forme d'un commun numérique est ainsi clairement affichée (voir Fig. 2).

Figure 2. Tweet d'Etalab à la signature officielle du partenariat

Etalab @Etalab · 15 avr. 2015

etalab Inauguration de la Base Adresse Nationale collaborative "I'adresse est vivante, nous inaugurons un bien commun" \#BAN

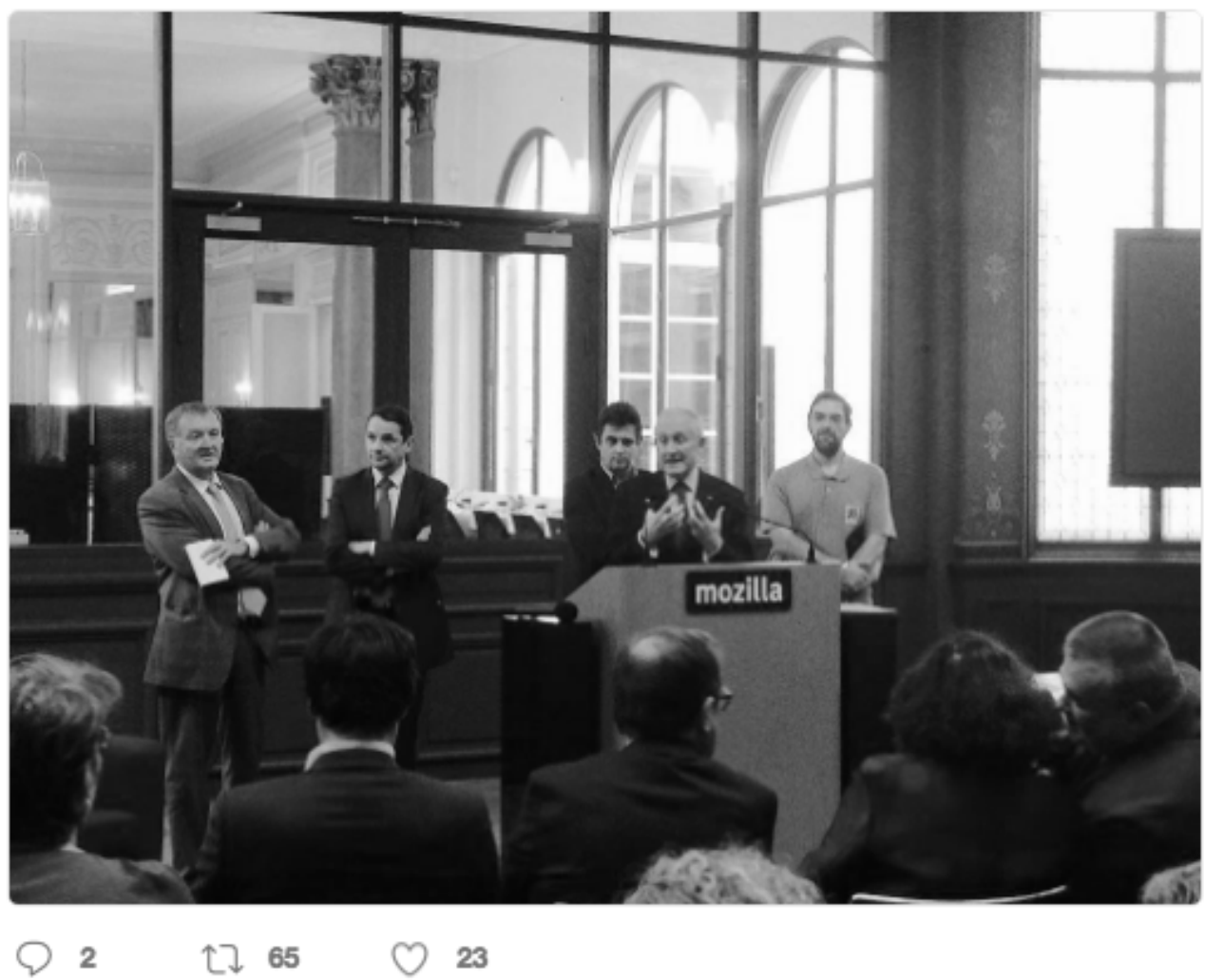

Lecture : de gauche à droite, Daniel Burseau (directeur de l'IGN), Thierry Mandon (secrétaire d'État à la Réforme de l'État et à la Simplification), Henri Verdier, Philippe Wahl (directeur générale de La Poste) et Christian Quest (porte-parole d'OSM). 
Nous avons vu comment le problème public de la production et de la diffusion d'une base adresse nationale est étatisé d'une manière tout à fait singulière. Dans le contexte d'une forte attente de l'État, un nouvel acteur (OSM) vient déstabiliser l'ordre administratif en produisant un commun numérique. Un entrepreneur de réforme (H. Verdier) se saisit de l'occasion pour mener à bien la réforme de l'État qu'il envisage en adoptant la forme sociotechnique du commun numérique. Cette forme s'adapte bien au discours réformateur qu'il porte, et permet d'enrôler tous les acteurs.

Si la plasticité du discours réformateur a permis la signature d'un partenariat original, elle n'a pas empêché les résistances institutionnelles d'émerger. La forme du commun numérique a-telle "infusée" dans l'administration et changée la relation entre l'État et les citoyens en redonnant du pouvoir d'agir à ces derniers ? Il nous faut maintenant relater les épreuves soulevées par cette forme du commun au sein de la machinerie étatique.

\section{LES RÉSISTANCES INSTITUTIONNELLES AUX COMMUNS NUMÉRIQUES}

Dans cette partie, je montre que les différentes caractéristiques des communs numériques qui peuvent potentiellement reconfigurer la relation État/citoyens (Linders, 2012), en particulier la contribution citoyenne à l'enrichissement d'une ressource informationnelle officielle, l'auto-organisation et le libre accès ont soulevé des résistances de la part des bénéficiaires du système institutionnel en place.

\section{L'enjeu du statut face au citizen sourcing}

Déjà avant la présignature de 2014, une note de la représentante du personnel CGT de l'IGN s'indignait du soutien du SGMAP à la BANO produite par de «simples citoyens » sans mandat officiel. Elle s'y interroge : " C'est quoi, la fameuse 'donnée faisant autorité' dès lors que l'État ne s'appuie pas sur 'son' opérateur national pour la constituer ?!» Elle écrit un mois plus tard une demande d'audience officielle au Premier ministre où elle explique que cette situation « remet en cause directement [1]es missions [de l'IGN] ». Pierre Pezziardi, un des premiers membres d'Etalab, écrit à ce propos dans un double entretien avec H. Verdier: «Pour les cartographes de l'IGN, il fallait accepter de ne plus être les producteurs primaires de l'information et cesser de considérer avec hostilité les autres intervenants du secteur ». La première résistance a donc concerné la légitimité administrative de certains acteurs à produire l'information géographique officielle.

La question, qui s'est posée à de nombreuses réunions de travail et publiques auxquelles j'ai assisté, était de savoir quel statut donner à la contribution de citoyens précisément sans statut ou mandat particuliers. Plusieurs critiques ont été soulevées.

"la contribution, ça ne marche que sur 3\% du territoire. (...) [En] zone dense, effectivement Openstreetmap sera meilleur que l'IGN, c'est évident. Face à 10.000 contributeurs, on ne peut rien parce que c'est à jour, et là la multitude prend tout son sens. Mais il faut qu'il y ait une multitude. Et les 97\% restant ? (...) l'IGN depuis 350 ans a eu un métier qui consistait à acquérir des donner, les vérifier trois fois, les certifier et faire des cartes. Le croisement avec l'approche de la multitude, où si on a une erreur on considère que c'est bon parce que la multitude va la corriger, c'est un peu compliqué " (ancien directeur de l'IGN, juillet 2018)

"C. - et puis on ne sait pas qui fait les modifications ? Je me permets de dire que c'est important de faire de la traçabilité, de «sourcer » pour savoir qui a produit l'information. Je sais qu'OSM France veut faire du collaboratif, mais le problème vient toujours sur la qualité de l'adresse

E. - et la possibilité de la fraude... 
C. Oui c'est vrai. (...) Le problème du collaboratif, c'est que ça dilue la responsabilité" (échange entre C., chef de la cellule de géomatique de la Direction générale de la Gendarmerie nationale et E. responsable de la BAN à l'IGN lors d'un atelier BAN dans les locaux de l'IGN, novembre 2017)

Égalité et qualité du service public, risques de fraude, dilution de la responsabilité sont trois critiques, propres à des acteurs publics, contre la contribution citoyenne à la BAN. L'architecture de la BAN (qui a aujourd'hui changé nous le verrons à la partie suivante) reposait sur la collection de diverses sources «de confiance» de données adresses faisant autorité (voir Fig. 3). Dans la plupart des communs numériques, les modalités de contribution à la ressource sont déterminées par la régulation du contenu de la contribution et beaucoup plus rarement sur un statut officiel du contributeur (Cardon et Levrel, 2009 ; Fuster Morell, 2014). Dans le cas de la BAN, au lieu de réguler le citizen sourcing, les acteurs ont privilégié de réguler le statut des contributeurs, en l'occurrence ici des entités officielles ou reconnues.

Figure 3. : les sources qui alimentaient la BAN

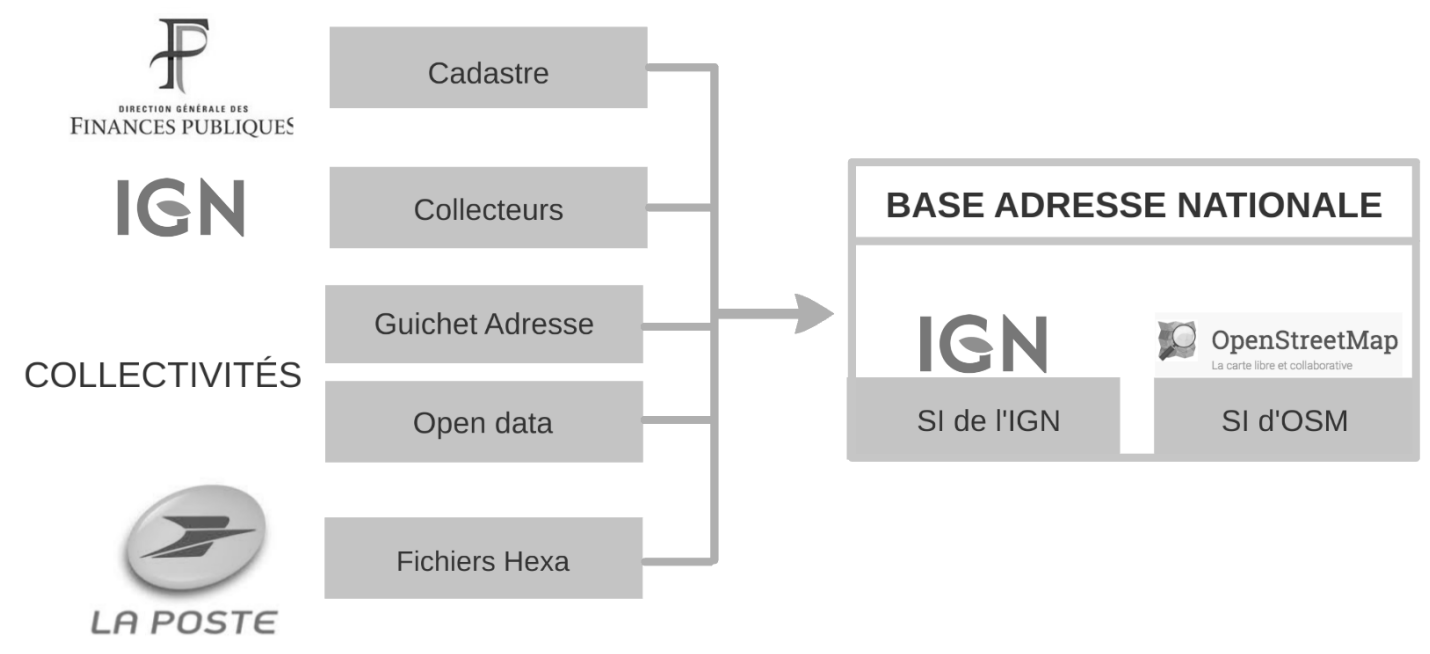

Lecture : Les différentes sources - DG Finances publiques, IGN, collectivités, La Poste - alimentant la BAN avant le 1" janvier 2020

\section{Do-it-yourself vs hiérarchie : deux éthos se confrontent}

«C'était une vraie gageure de faire travailler des gens de mondes différents. C'est un peu la difficulté entre le monde de l'Internet agile qui peut bouger très vite, et l'IGN avec des grosses structures. En même temps il faut faire du souverain, et en même temps il faut travailler avec des jeunes chevelus barbus, vous voyez le changement de culture » (ancien directeur de l'IGN, juillet 2018).

Ce témoignage de l'ancien directeur de l'IGN résume bien une situation de résistance organisationnelle. La gouvernance de la BAN, qui a prévalu entre 2015 et 2020, comportait deux strates. La première, le comité de pilotage, était composée des différentes directions et se réunissait théoriquement tous les six mois pour décider des grandes orientations (juridiques, économiques, etc.). La seconde, le comité technique, était composée des ingénieurs et développeurs censés mettre en œuvre les décisions du comité de pilotage. Ce mode de fonctionnement où chaque niveau hiérarchique collabore avec leurs pairs dans les autres administrations est caractéristique de la culture administrative. Mais il entre en contradiction avec le principe de l'autogouvernement des communs numériques et plus généralement avec les pratiques et l'éthos d'une partie des hackers (Himanen, 2001 ; Khalil, 2011). Comme en témoignent respectivement le président d'OSM France et le responsable de la BAN à Etalab : 
«Etalab et à la fois OSM qui sont culturellement sur un fonctionnement horizontal (...) on cherche à faire simple, direct, par nous-même. Et ça, c'est quelque chose d'assez fort (...) par rapport à la culture d'entreprise de La poste et IGN la culture de l'État.» (novembre 2017, je souligne)

«Moi j'ai proposé plusieurs fois qu'on avance sur des sujets juridiques, des modèles économiques, faire des ateliers ensemble, proposer des choses à nos «chefs », mais ils ne veulent pas proposer des choses qui sortent de leur rôle. » (responsable de la BAN à Etalab, juin 2018).

Un entretien mené avec la directrice technique de la BAN à l'IGN semble confirmer cette dernière affirmation. Quand je lui demande la manière dont se déroule la discussion autour des licences, elle me répond: "Moi ça ne me concerne pas. On me demande de faire des choses techniques je les fais, mais les licences pour moi ce n'est pas un problème. (...) C'est un truc de chefs et de juristes. » (novembre 2017).

La promesse d'auto-organisation de la $\mathrm{BAN}$ en tant que commun numérique, où des agents étatiques et non étatiques auraient pu indistinctement collaborer de manière "horizontale" est ainsi venue buter contre une culture administrative hiérarchique ancrée dans les pratiques des agents. Cette résistance organisationnelle impliquait d'avoir l'aval politique avant toute prise de décision stratégique, à l'inverse de modes de gouvernance plus souples de certains projets de logiciels libres qui mélangent subtilement hiérarchie et do-ocratie (Demazière, Horn et Zune, 2007 ; O’Mahony et Ferraro, 2007 ; Schweik et English, 2012).

\section{Government as a plateforme pour ou contre Google? Une question juridico-économique}

Au-delà de ces deux résistances, la question de savoir qui pouvait y accéder et selon quelles conditions - ou dans nos termes quelles modalités pour un État-plateforme - a été le point d'achoppement le plus problématique. Pour comprendre cette situation, il faut revenir un instant en arrière pour étudier l'évolution de la situation des bénéficiaires de l'ancienne situation institutionnelle avant la mise en place de la BAN.

Historiquement, l'IGN vendait des cartes papier car les dotations de l'État ne représentent que $60 \%$ de son budget total. Or entre les années 2000 et 2010, ses ventes papier chute et cet institut se repositionne sur la vente de bases de données numériques. Les recettes générées par la $\mathrm{BD}$ adresse numérique, vendue principalement à de grandes entreprises comme Google, représentent quelques millions d'euros par an. Mais les années 2010 voient l'émergence d'exigences légales d'ouverture des données publiques qui obligent l'IGN à rendre disponibles gratuitement certaines bases de données. Ces contraintes légales ambivalentes génèrent des contraintes budgétaires fortes sur l'institution ${ }^{2 s}$.

Durant la même décennie 2000, La poste subit la baisse du volume de courrier et l'ouverture à la concurrence, un an après avoir été transformée en société anonyme à capitaux publics. La vente des services liés à leurs bases de données adresse, générant autour de cinq millions d'euros, vient combler en partie ces pertes. L'IGN et La Poste collaborent pour réaliser certains échanges d'information sur leurs données respectives «dans une logique commerciale » (entretien avec Pascal Bartier, directeur des ventes chez La Poste, novembre 2018)..

Cette tension économique, qui explique pourquoi ces acteurs étaient réticents à voir advenir une BAN ouverte et gratuite, s'est faite ressentir autour de la licence de la BAN. Elle est étroitement liée à la question juridique de propriété. Qui possède la BAN, qui peut l'exploiter

${ }^{28}$ Voir notamment la baisse de dotation de plusieurs millions d'euros suite à la décision de rendre le RGE gratuit pour les acteurs publics. Source : Rapport d'activité 2018 de l'IGN (2018). 
et selon quelles conditions ? Les réponses à ces questions déterminent non seulement la possibilité de mener une activité commerciale autour de la BAN, mais également le financement de cette base et le rôle informationnel de l'administration vis-à-vis du marché et de la société : en bref, elles ont un impact sur ce qui relève de l'État et de la manière d'organiser ce dernier (Bezes et Siné, 2011).

Les débats autour de la propriété de la BAN se sont cristallisés sur le choix des licences". À la signature du partenariat en 2015, deux licences sont prévues : OSM la diffuserait sous licence Open Database Licence et Etalab sous la licence gratuite de repartage. La licence Ouverte, habituellement utilisée par Etalab, n'est pas retenue (voir encadré 3.)

Encadré 3. - Trois licences pour une BAN

- Open Database Licence (ODbL) - Cette licence issue du projet opendatacommons.org de l'Open Knowledge Foundation est largement utilisée dans le monde de l'open data. Elle autorise tout type d'utilisation (consultation, téléchargement, services commerciaux, etc.) à condition de maintenir la base de données (même modifiée) toujours ouverte et explicitement sous licence OdbL. C'est la raison pour laquelle on dit qu'elle est «contaminante ». Elle permet ainsi d'éviter tout type «d'enclosure » sur une base de données constituée ainsi en bien commun accessible à tous.

- Licence Gratuit de repartage - Cette licence ad hoc est écrite par les juristes de La Poste et de l'IGN. Elle stipule que la base de données est gratuitement exploitable par tous les acteurs publics, non lucratifs ou ayant un usage personnel. Ce qui implique que les acteurs économiques en sont exclus et qu'ils devront utiliser les offres commerciales (de La Poste ou de l'IGN). Elle indique aussi que les « fondateurs » restent «propriétaires » de la BAN, et que les utilisateurs doivent leur reverser l'ensemble des enrichissements.

- Licence Ouverte - Cette licence est la plus permissive de toutes puisqu'elle autorise n'importe quel usage des données, même la possibilité de les enclore et de les vendre, à la seule condition pour l'utilisateur d'en mentionner la paternité.

Dès l'annonce de la signature du partenariat, la seconde licence est vivement critiquée par les juristes de l'association savoirscom1, qui promeut les biens communs depuis sa création en 2011. Pour eux, cette licence n'est compatible « ni dans l'esprit ni en pratique avec la notion d'Open Data - et encore moins de Biens Communs Numériques » (SavoirsCom1, 2015). La critique porte sur le fait que La Poste et l'IGN restent en quelque sorte propriétaires de la BAN et peuvent unilatéralement résilier son accès, sans compter qu'ils en excluent tous les acteurs économiques sans distinction. Or pour l'ancien directeur de l'IGN, c'était là tout l'intérêt :

« Ok on met à disposition des données, mais comment ne pas faire le lit des GAFA. Moi je paie mes impôts en France, je veux bien créer de la valeur ajoutée à Google, mais ça a une limite. Donc on s'en était sorti en faisant une licence gratuite de repartage, et les gens qui ne veulent pas repartager, quand c'est Google qui aspire la donnée sans contribuer, tant pis ils paient » (juillet 2018).

On peut comprendre l'étonnement de savoirsCom1 en rappelant que l'action d'Etalab en général et d'H. Verdier en particulier était tournée vers un open data sans restriction. Cette orientation aurait dû a priori faire tendre la BAN vers la licence Ouverte. Pourquoi cette licence n'a donc pas été choisie dans le partenariat? H. Verdier raconte :

«Christian [Quest] m'a dit que «le problème c'est que la licence ouverte c'est une licence trop libérale ». J'ai dit «c'est bien non ? On fait de l'open data, on veut le plus d'usage possible pour le plus de gens possible. » Il me dit «oui, mais pas là. Voilà ce qui va se passer. On a une base juste à $85 \%$. Les contributeurs BANO vont venir t'aider, elle va devenir juste à $95 \%$. Google va tout ramasser pour la rendre juste à $98 \%$ et va prendre $100 \%$ des usages parce qu'ils auront une base plus complète, des meilleurs API et les datacenters les plus performants. Tout le monde ira chez eux.»

"Pour une synthèse sur la propriété des données publiques, voir (Camus, 2018) 
(...) Et il m'a expliqué la licence ODbL, la licence contaminante, le share alike. C'est après que j'ai compris que c'était un cas pur de commun. Pour la licence gratuite de repartage, je trouvais ça génial que ceux qui utilisaient la ressource étaient contraints d'enrichir la ressource (...). Je me suis rendu compte qu'il y avait un open data étatiste et un open data libéral"

On touche dans ces débats à un point crucial. Pour comprendre la manière dont les acteurs orientent leurs actions, il faut comprendre la manière dont ils les justifient à la lumière de grands principes généraux concernant l'État, notamment son rapport au marché. Ici, trois conceptions antagonistes de la BAN se dessinent.

Pour le responsable de la BAN chez Etalab ainsi que la députée ayant écrit un rapport sur la question, la BAN est une ressource produite et gérée par l'État, qui doit être financée par l'impôt, dont l'accès et l'usage sont garantis et gratuits pour tous, même les GAFA". Cette conception, inscrite dans la licence Ouvert, est portée par les promoteurs de l'open data et plus généralement par les partisans d'un État-plateforme pro-marché qui réfute toute forme de "protectionnisme" informationnelis. Pour OSM et Savoircom1, la BAN est une ressource d'intérêt général dont l'accès et l'usage doivent être garantis à tous, et protégée contre toute forme de réappropriation privative. Cette conception est inscrite dans la licence ODbL. Pour l'IGN et $\mathrm{H}$. Verdier, la BAN est une ressource produite par une communauté qui souhaite éviter sa réappropriation, mais surtout l'enrichir de manière pérenne en évitant les passagers clandestins. Si Google ne paie pas ses impôts en France et ne souhaite pas contribuer à la BAN, et bien qu'ils y contribuent financièrement en payant pour y avoir accès. Cette vision était portée par la Licence gratuite de repartage. Proche de la vision d'OSM, la clause stipulant que la BAN restait la propriété de La Poste et de l'IGN a été cependant l'objet d'un désaccord de principe.

Tableau 2. Récapitulatif

\begin{tabular}{|c|c|c|c|}
\hline Acteurs & Revendication & Licence & Figure d'État \\
\hline $\begin{array}{l}\text { Etalab, } \\
\text { députée }\end{array}$ & $\begin{array}{l}\text { La BAN doit être librement accessible par tous } \\
\text { les acteurs sans condition, même Google }\end{array}$ & $\begin{array}{l}\text { Licence } \\
\text { Ouverte }\end{array}$ & $\begin{array}{l}\text { Etat plateforme « pro- } \\
\text { marché » }\end{array}$ \\
\hline $\begin{array}{c}\text { OSM, } \\
\text { Savoircom } \\
1\end{array}$ & $\begin{array}{l}\text { La BAN doit être librement accessible par tous } \\
\text { les acteurs, mais protégée contre les } \\
\text { réappropriations exclusives de Google }\end{array}$ & $\begin{array}{l}\text { Licence } \\
\text { OdBL }\end{array}$ & $\begin{array}{c}\text { État plateforme } \\
\text { «protectionniste » }\end{array}$ \\
\hline $\begin{array}{l}\text { IGN, H. } \\
\text { Verdier }\end{array}$ & $\begin{array}{l}\text { La BAN doit être librement accessible, } \\
\text { protégée contre les réappropriations exclusives } \\
\text { et les passagers clandestins comme Google }\end{array}$ & $\begin{array}{l}\text { Licence } \\
\text { gratuite de } \\
\text { repartage }\end{array}$ & État plateforme « étatiste » \\
\hline
\end{tabular}

La forme générale du commun numérique comme composante d'un projet de réforme de l'État a ainsi trouvé sa limite dans la concrétisation de ses multiples définitions. Fin 2017, l'ensemble de ces résistances - de légitimité, organisationnelle, et juridico-économique - ont conduit le projet de la BAN au point mort. Entre fin 2017 et fin 2019, aucun comité de pilotage ne se réunit. La BAN est diffusée à titre temporaire par l'IGN sous la licence gratuit de repartage qui devient caduque fin 2018, alors que OSM continue à diffuser la première base créée, la BANO, sous licence ODbL. L'actuel président d'OSM France me résume la situation : «Dans le meilleur des cas flottement, sinon... heu, mort clinique ou abandon, c'est radical, mais en l'absence totale de fonctionnement du copil [comité de pilotage], ça ne m'inspire rien d'autre. » (octobre 2019).

\footnotetext{
${ }^{3}$ Notons que la gratuité de l'information publique fait débat au sein de l'administration depuis la publication du rapport Levy-Jouyet sur «L'économie de l'immatériel, la croissance de demain » (2007).

${ }^{35}$ Comme le stipule l'actuelle FAQ du site de la BAN : " seule la Licence Ouverte permet d'alimenter la Base Adresse Nationale, le cadastre, l'INSEE et les principales solutions cartographiques et GPS du marché. Si une forme de protectionnisme peut être pertinente sur certaines données publiques, elle est exclue sur les adresses qui constituent une donnée fondamentale". Source : https://adresse.data.gouv.fr/faq
} 
Pourtant, la BAN est aujourd'hui produite par l'État et diffusée en licence Ouverte. Dès lors, pourquoi la forme du commun numérique a-t-elle été abandonnée au profit d'une vision plus libérale de l'open data et de l'État-plateforme?

\section{L’ABANDON DE LA FORME DU COMMUN NUMÉRIQUE}

Trois facteurs peuvent expliquer ce changement. La difficulté à dépasser les résistances institutionnelles à l'adoption de la forme du commun numérique dont j'ai parlé. L'alternance politique de 2017 entrainant le remerciement d'H. Verdier, la démission de C. Quest et l'arrivée au pouvoir d'un personnel politique porteur d'une vision libérale de l'open data. La création d'un service public de la donnée qui «normalise » la production de la BAN relevant ainsi d'une mission étatique et souveraine.

\section{L'impact du changement du personnel politique}

Le changement de majorité de 2017 entraine un certain nombre de conséquences au sein de l'administration en générale, et pour l'action d'Etalab en particulier. D'une part, un réseau interpersonnel avait été créé entre les membres d'Etalab et les différentes entités (cabinets ministeriels, de l'Elysée, etc.) et sa structuration s'est affaiblie à l'arrivée d'un nouveau personnel politique. D'autre part, les représentations concernant le rôle du numérique pour réformer l'État ont divergés. Nadi Bou Hanna remplace Henri Verdier à la tête de la DINSIC. Ce polytechnicien insiste dans ses discours et ses actions sur l'importance de la modernisation interne de l'infrastructure des SI de l'État plutôt que sur la transformation numérique de la relation État/citoyens et «l'ouverture» de l'administration. Cette divergence de vision est critiquée par plusieurs membres historiques de la DINSIC qui démissionnent, dont C. Quest. Voici un extrait de sa lettre de démission :

« Cette mission rattachée au premier ministre était à ma connaissance une des rares administrations réellement ouverte sur le monde extérieur. (...) Votre feuille de route [Nadi Bou Hanna] utilitariste et court-termiste n'intégrant quasiment aucune des valeurs qui m'ont fait rejoindre Etalab (...) m'oblige à prendre une autre route.»

Finalement, le concept de « communs numériques » ne s'inscrit ni dans la tradition ni dans les discours politiques de la nouvelle majoritée. Pour essayer de débloquer la situation de la BAN, une députée du groupe En Marche! est appelée à publier un rapport sur Les données géographiques souveraines qui paraît fin 2018. Cette dernière a travaillé dans le secteur de l'assurance avant se députation et se qualifie de «libérale de droite économiquement» (entretien, avril 2019) . Dans son rapport, elle préconise pour remédier aux difficultés concernant la BAN de la considérer comme « un bien public », produit de manière souveraine par l'administration, financé par l'impôt, et publié sous licence Ouverte. (Faure-Muntian, 2018). Ces choix renvoient à la perspective d'un État plateforme «pro-marché » tel que je l'ai défini (voir tableau 2.). Lorsque je lui demande si elle est famillière avec le concept de « communs numériques » elle me répondons que : « ce n'est pas le terme le plus utilisé (...) et il ne s'agit d'une priorité pour la majorité. (...) Nous on a tendance à l'utiliser surtout dans la perspective de l'open data et de l'Etat plateforme » (entretien, avril 2019).

Suite à ce rapport, un référé est envoyé au Premier ministre par la Cour des comptes pour éclaircir la situation de la BAN. Une demande formelle est adressée au Premier ministre pour qu'il mette fin au blocage. Cette réponse publiée par le cabinet du Premier ministre en mars 2019 va dans le sens des conclusions de la députée de sa majorité :

"Je considère que la constitution d'une base adresse nationale gratuite et unifiée est un enjeu important pour l'économie française ainsi que pour la chaîne de décision publique (...). J'ai demandé à la DINSIC en lien avec les acteurs concernés de mettre en œuvre de nouvelles modalités de gouvernance et de fonctionnement, reposant sur la gratuité et en même temps sur la qualité de mise à jour collaborative, afin que la 
BAN soit effectivement diffusée gratuitement (...) au plus tard au 1er janvier 2020 sous licence ouverte." (Philippe, 2019).

Le texte indique ainsi que l'État doit prendre en charge lui-même le fonctionnement de la BAN et la DINSIC est appelée à restructurer la gouvernance pour créer une collaboration au sein de l'administration. Au cours de cette même période, la production et la diffusion de la BAN sont promues au rang de service public. La Loi Lemaire, dont les décrets d'application sont publiés en 2017, crée le service public de la donnée. Ce dernier vise à mettre à disposition neuf jeux de données de référence «en vue de faciliter leur réutilisation ${ }^{* 0}$ » dont la BAN. Comme l'indique Cluzel-Métayer, le choix d'ériger une activité en service public « est significatif d'une volonté de la puissance publique d'assumer la maîtrise de la satisfaction d'un besoin qu'elle considère d'intérêt général et pour lequel elle juge l'initiative privée sinon totalement, au moins partiellement inadaptée »(Cluzel-Métayer, 2018). La BAN est ainsi inscrite dans la loi en tant que base de données prise en charge par la puissance publique. Pour le nouveau personnel politique, l'expérimentation de nouvelles formes d'interactions entre État et citoyens à travers des formes de co-production d'informations publics est ainsi abandonnée pour une vision plus pro-marché d'un État-plateforme producteur souverain d'une information diffusée gratuitement en open data.

\section{L'abandon de deux caractéristiques de la forme du commun numérique}

Depuis ce texte, le responsable DINSIC de la BAN se place sous l'autorité du Premier ministre pour aller discuter de nouveau avec l'ensemble des acteurs publics et remettre la gouvernance à plat. Bien que la gouvernance ne soit pas encore stabilisée à ce jour, plusieurs choses sont à noter.

D'une part, OSM s'éloigne de la gouvernance surtout depuis le départ de C. Quest de la DINSIC. Les membres d'OSM relancent les discussions pour améliorer la BANO, cette base alternative à celle de l'État qui avait engagée l'épreuve. La nouvelle gouvernance de la BAN, qui n'est coordonnée que par un acteur, prévoit une strate opérationnelle avec deux acteurs publics, l'IGN et la DINSIC, un collège regroupant les contributeurs nationaux reconnus (comme l'INSEE) et "un lieu de discussion général avec les réutilisateurs généraux". Finalement, le travail entre l'IGN et la DINSIC se fait à distance. Comme le relate le responsable de la BAN à la DISNIC : «Avec l'IGN on ne travaille plus ensemble, on n'a pas les mêmes méthodes de travail (...) on travaille sur nos briques et ça marche mieux comme ça.» (octobre 2019). Bien que la gouvernance ne soit pas encore stabilisée, elle semble s'éloigner de la caractéristique d'autogouvernement propre aux communs numériques.

La contribution citoyenne a disparu du fil des discussions. Sans être définitivement écartée, la mise en place d'un dispositif technique qui la rendrait possible est pour l'heure ajournée. D'autre part, les contributions volontaires à la BANO via OSM ne pourront plus être insérées dans la BAN à cause de l'impossibilité juridique d'intégrer des données sous licence ODbL à une base de données sous licence Ouverte.

\section{Figure 4. Comment contribuer à la BAN}

\section{En tant que citoyen}

II n'existe pas encore de dispositif national permettant aux citoyens de contribuer directement, mais de nombreux guichets de signalement existent à l'échelon local. Ce site a vocation à les référencer à moyen terme.

En attendant, contactez votre mairie ou votre EPCI, et parlez-leur de nous !

Notice : imprimé-écran du site de la BAN, à la section " contribuer à la BAN". source : adresse.data.gouv.fr, consulté le 10/03/2020

\footnotetext{
${ }^{\star}$ Source : www.economie.gouv.fr/republique-numerique
} 
Concernant le choix de la licence Ouvert, elle indique que les acteurs publics ont privilégié la perspective d'un État-plateforme pro-marché qui produit et diffuse gratuitement un bien informationnel public dans le cadre d'un service public. L'État, en endossant son rôle d'Étatplateforme, investit dans une infrastructure informationnelle qui sert tous types d'acteurs, dont ceux du secteur privé. Comme le préconise le techno-optimiste T. O'Reilly, inventeur du concept de government as a platform :

"Investing in infrastructure (...) will lead to a more robust private sector ecosystem. (...) The whole point of government as a platform is to encourage the private sector to build applications that government didn't consider or doesn't have the resources to create (O'Reilly, 2010)"

Les deux caractéristiques des communs numériques que sont l'autogouvernement et la contribution des utilisateurs à l'enrichissement de la ressource semblent avoir été abandonnées. La caractéristique du libre accès est maintenue, mais sans garantie contre un risque de réappropriation et de passager clandestin de la part des GAFAM. Il est d'ailleurs notable que dans le référé du Premier ministre, le terme de commun numérique soit absent.

\section{CONCLUSION}

Deux énigmes ont traversé mon enquête : pourquoi la forme sociotechnique du commun numérique a-t-elle été adoptée pour créer la BAN, et qu'est-ce que cette forme est venue déplacer dans la relation État/société ? Je voulais également tester l'hypothèse de «l'infusion institutionnelle ».

J'ai répondu à la première question en expliquant que la forme sociotechnique du commun numérique a été adoptée pour tenter de réformer l'État. Elle l'a été dans un contexte particulier, celui de l'émergence d'OSM et de l'action de C. Quest, représentant le courant des communs numériques, dans le problème public de l'adresse. Elle traduisait à la fois la perspective réformatrice d'H. Verdier et les intérêts des différents acteurs, ce qui a permis d'aboutir à la signature du partenariat. Mais cette forme du commun numérique a rencontré des résistances institutionnelles, car elle remettait en cause la légitimité, l'organisation et les rentes financières des acteurs qui bénéficiaient de l'ordre institutionnel précédent. Sa mise en place a généré de profonds débats sur le rôle et le mode d'organisation de l'État.

Est-ce que, finalement, quelque chose a été déplacé dans la frontière État/société ? La forme du commun numérique a-t-elle infusé dans l'administration en distribuant le pouvoir de décider la production et l'usage d'une information officielle ? Parmi les différentes options d'interaction entre État et société que pouvait préfigurer l'adoption de la forme du commun numérique par l'État, le citizen sourcing et le do-it-yourself government semblent avoir été abandonnées au profit d'un type d'État-plateforme. Ainsi, seul le critère de l'accès à la ressource, qui n'était pas possible avant la BAN, semble avoir "infusé" grâce aux actions d'acteurs multipositionnés comme C. Quest et H. Verdier. Mais le choix de la licence Ouverte au moment de l'arrivée d'un nouveau personnel politique témoigne du fait que la ressource n'est pas protégée contre les risques de réappropriation exclusive. Le but n'est pas comme avec les licences OdBL ou Gratuit de repartage de se prémunir des GAFA, mais de les renforcer, au risque de ne donner du pouvoir informationnel qu'à ceux qui en ont déjà (Badouard, 2015; Goëta et Mabi, 2014 ; Gurstein, 2011). À ceci près que l'État reste souverain en gardant le monopole de la production de la BAN. L'abandon de la forme du commun numérique dans la structure socio-technique de la BAN témoigne ainsi du fait que l'État met en ouvre une forme particulière d'exercice du pouvoir informationnel en empêchant techniquement la contribution citoyenne et en favorisant juridiquement sa réutilisation par des acteurs économiques. Le numérique, loin de conduire obligatoirement à un désengagement néolibéral de l'État ou à sa démocratisation, ouvert grâce à lui à la force de la "multitude", est un espace où peut au contraire s'affirmer un «État informationnel ». Cet État, nous dit Braman, exerce une forme particulière de pouvoir à travers la maitrise de la 
production et la diffusion d'informations importantes et largement utilisées dans la société (Braman, 2009). L'étude de la BAN en tant que dispositif qui permet, contraint ou favorise certains acteurs et certaines pratiques, nous a permis de mettre à jour les caractéristiques d'une direction de la transformation numérique de l'État et de sa relation à la société, qui prend ici la forme d'un État-plateforme souverain et pro-marché.

La limite principale de notre travail tient à la contemporanéité de notre objet. Les évolutions de la BAN sont rapides, et il est possible que des effets "d'infusion institutionnelle" et partant, que la reconfiguration de la relation État/citoyen, ne se soient pas encore pleinement déployées. Ainsi la contribution citoyenne à la BAN semble aujourd'hui possible par des voies détournées (Merlière, 2019) et cette contribution s'institutionnalisera peut-être. Il faudra donc être attentif aux évolutions de la BAN ainsi qu'à d'autres expérimentations de communs numériques mis en place par l'État (Shulz, 2019) pour saisir in concreto les reconfigurations numériques en cours de ce dernier.

\section{Bibliographie}

AFIGEO, 2010, « Atelier national sur les Données Adresse », Compte rendu, Paris. AKrich, M., Callon, M., LATOur, B. (dirs.), 2013, Sociologie de la traduction: Textes fondateurs, Paris, Presses des Mines (Sciences sociales), $401 \mathrm{p}$.

AlAuZEN M., 2019, Plis et replis de l'État plateforme. Enquête sur la modernisation des services publics en France, thesis, Paris Sciences et Lettres.

ARI-VeIKKo A., 2008, Electronic Government: Concepts, Methodologies, Tools, and Applications, New York: Information Science Reference, New York, IGI Global, 4336 p. BADOUARD R., 2015, « Open Government, open data : l'empowerment citoyen en question », dans Open data, Big data, Sciences des données, Editions de la MSH.

BATES J., 2014, « The strategic importance of information policy for the contemporary neoliberal state: the case of Open Government Data in the United Kingdom », Government Information Quarterly, 31, 3, p. 388395.

Beauvallet G., Ronai M., 2005, «Vivre a temps réels », Reseaux, $n^{\circ}$ 129-130, 1, p. 275309.

BELLON A., 2018, Gouverner l'internet. Mobilisations, expertises et bureaucraties dans la fabrique des politiques numériques (1969-2017), Thèse de doctorat, Paris, Université Paris 1 - Panthéon-Sorbonne.

BEZES P., 2009, Réinventer l'État: les réformes de l'administration française (1962 - 2008), Paris, Presses Universitaires de France - PUF, 544 p.

BEZES P., LE LIDEC P., 2011, «Ordre institutionnel et genèse des réformes », dans Sociologie de l'institution, Paris, BELIN LITTERATURE ET REVUES, p. 5575.

BEZES P., SINÉ A., 2011, « Gouverner (par) les finances publiques : introduction », dans Gouverner (par) les finances publiques, Paris, Presses de Sciences Po (P.F.N.S.P.), p. 17111.

BOLTANSKI L., 2012, Énigmes et complots: une enquête à propos d'enquêtes, Paris, Gallimard (NRF essais), $461 \mathrm{p}$.

Bourdieu P., 2015, Sur l'état. Cours au collège de France, Paris, Points, 720 p.

BRAMAn S., 2009, Change of state: information, policy, and power, Cambridge, Mass.; London, Eng., The Mit Press.

BRÉTHAUT C., NAHRATH S., 2010, « Entre imbrication, instrumentalisation et infusion: le rôle des consortages de bisses et des bourgeoisies dans les politiques de gestion de l'eau à Crans-Montana »,.

CAMUS A., 2018, « La propriété des données publiques », Revue francaise d'administration publique, $N^{\circ} 167,3$, p. 479490.

CARDON D., LEVREL J., 2009, « La vigilance participative. Une interprétation de la gouvernance de Wikipédia », Réseaux, 154, p. 5189.

CHIGNARD S., BENYAYER L.-D., 2015, Datanomics Les nouveaux business models des 
données, Limoges, FYP EDITIONS, 192 p.

CluZEL-MÉTAYER L., 2018, "La construction d'un service public de la donnée », Revue francaise d'administration publique, $N^{\circ} 167,3$, p. 491500.

DeMAZIÈRE D., HoRn F., ZunE M., 2007, « Des relations de travail sans règles ? », Societes contemporaines, $n^{\circ} 66,2$, p. 101125.

DENĖGRE J., SALGÉ F., 2004, « Introduction aux systèmes d'information géographique », dans Que sais-je?,p. 511.

DufÉAl M., JONChÈres C., Noucher M., 2016, « Ecce Carto - Des espaces de la contribution à la contribution sur l'Espace - Profils, pratiques et valeurs d'engagement des contributeurs d'OpenStreetMap (OSM) », report, UMR 5319.

DufÉAL M., Noucher M., 2017, « Des TIC au TOC. Contribuer à OpenStreetMap : entre commun numérique et utopie cartographique », Netcom. Réseaux, communication et territoires, 31-1/2, p. 7798.

FAURE-MUNTIAN V., 2018, « Les données géographiques souveraines », Rapport au gouvernement, Assemblée nationale.

FRANCESCHETTI L., 2016, « The Open Government policy as a strategic use of information to entrench neoliberalism? The case of Italy », Partecipazione e conflitto, 9, 2.

FUSTER MORELl M., 2014, « Governance of Online Creation Communities for the Building of Digital Commons: Viewed Through the Framework of the Institutional Analysis and Development », dans Governing Knowledge Commons, Oxford University Press, Rochester, NY, Social Science Research Network.

GARDEY D., 2008, Écrire, calculer, classer, La Découverte (anthropologie des sciences et des techniques).

GOËTA S., 2016, Instaurer des données, instaurer des publics Une enquête sociologique dans les coulisses de l'open data, Thèse de doctorat, Télécom ParisTech.

GOËTA S., MABI C., 2014, «L'open data peut-il (encore) servir les citoyens ? », Mouvements, 79, p. 8191.

GoODY J., 1979, La Raison graphique: la domestication de la pensée sauvage, traduit par BAZIN J., BENSA A., Paris, Les Editions de Minuit, 274 p.

Groupe DE TRAVAIL ADRESSE, 2011, « Pour une politique nationale de la donnée Adresse », AFIGEO.

GURSTEIN M.B., 2011, « Open data: Empowering the empowered or effective data use for everyone? », First Monday, 16, 2.

HaKlay M., Singleton A., PARKer C., 2008, « Web Mapping 2.0: The Neogeography of the GeoWeb », Geography Compass, 2, 6, p. 20112039.

HIMANEN P., 2001, L'Ethique Hacker et l'Esprit de l'ère de l'information, traduit par LEBLANC C., Paris, Exils, 219 p.

JACOBS F., 2012, « The First Google Maps War », Opinionator.

KHALIL C., 2011, Les méthodes « agiles » de management de projets informatiques : une analyse "par la pratique », science de gestion, Paris, Télécom ParisTech.

LASCOUMES P., 2004, « La Gouvernementalité : de la critique de l'État aux technologies du pouvoir », Le Portique. Revue de philosophie et de sciences humaines, 1314.

LASCOUMES P., 2007, « Gouverner par les cartes », Geneses, $n^{\circ}$ 68, 3, p. 23.

LasCoumes P., Le Galès P., 2012, Sociologie de l'action publique: Domaines et approches, 2e édition, Paris, Armand Colin, 128 p.

LINDERS D., 2012, «From e-government to we-government: Defining a typology for citizen coproduction in the age of social media », Government Information Quarterly, 29, 4, p. 446454 .

LiNHARDT D., 2009, «L'Etat et ses épreuves. Éléments d'une sociologie des agencements étatiques»,Clio@Thémis. Revue électroniqued'histoire du droit.

LINHARDT D., 2012, «Avant-propos : épreuves d’État. Une variation sur la définition wébérienne de l'État», Quaderni. Communication, technologies, pouvoir, 78, p. 522. LINHARDT D., 2018, «l'État en société », dans Etat et société politique : Approches sociologiques et philosophiques, Paris, Editions de l'Ecole des Hautes Etudes en Sciences Sociales, p. 6382. 
LIPS M., 2012, « E-Government is dead: Long live Public Administration 2.0 », Information Polity, 17, 3,4, p. 239250.

MERLIÈRE T., 2019, « Comment un simple citoyen peut-il contribuer à résoudre les problèmes d'adresse de sa commune? », Medium.

O'MAHONY S., FERRARO F., 2007, « The Emergence of Governance in an Open Source Community », Academy of Management Journal, 50, 5, p. 10791106.

O’REILly T., 2010, " Government as a plateform », dans Open Government: Collaboration, Transparency, and Participation in Practice, O'Reilly Media, Inc.

PARLEMENT EUROPÉEN, 2003, « DIRECTIVE 2003/98/CE DU PARLEMENT EUROPÉEN ET DU CONSEIL du 17 novembre 2003 concernant la réutilisation des informations du secteur public », 2003/98/CE.

Pelletier M., 2013, Les Cartes des Cassini : La science au service de l'Etat et des provinces, 2e édition, Paris, Comité des travaux historiques et scientifiques - CTHS, $400 \mathrm{p}$. PhILIPPE E., 2019, « Référé sur la valorisation des données de l'Institut national de l'information géographique et forestière (IGN), de Météo-France et du Centre d'études et d'expertise sur les risques, de l'environnement, la mobilité et l'aménagement (CEREMA): l'enjeu de l'ouverture des données publiques », Paris, France, Premier ministre.

PlanTIN J.-C., 2018, « Google Maps as Cartographic Infrastructure: From Participatory Mapmaking to Database Maintenance », International Journal of Communication, 12, p. $489-506$.

SAVOIRSCOM1, 2015, « Ouverture de la Base d'Adresses Nationale (BAN) : la fête gâchée par une « licence piège »"».

SCHWEIK C.M., ENGLISH R.C., 2012, Internet success: a study of open-source software commons, Cambridge, Mass, MIT Press, $351 \mathrm{p}$.

SHULZ S., 2019, «Un logiciel libre pour lutter contre l'opacité du système sociofiscal », Revue francaise de science politique, Vol. 69, 5, p. 845868.

STAUB J., 2011, « Les frontières dans Google Maps, un enjeu géopolitique », Lettre d'information géomatique, avril 2011.

VERDIER P. PAR H., 2018, « Pour une administration libérée »,.

WeSt D.M., 2005, Digital Government: Technology and Public Sector Performance, Princeton University Press. 